\title{
Compression bandages or stockings versus no compression for treating venous leg ulcers (Protocol)
}

DOI:

10.1002/14651858.CD013397

\section{Document Version}

Final published version

Link to publication record in Manchester Research Explorer

\section{Citation for published version (APA):}

Shi, C., Dumville, J., \& Cullum, N. (2019). Compression bandages or stockings versus no compression for treating venous leg ulcers (Protocol). Cochrane Database of Systemic Reviews.

https://doi.org/10.1002/14651858.CD013397

\section{Published in:}

Cochrane Database of Systemic Reviews

\section{Citing this paper}

Please note that where the full-text provided on Manchester Research Explorer is the Author Accepted Manuscript or Proof version this may differ from the final Published version. If citing, it is advised that you check and use the publisher's definitive version.

\section{General rights}

Copyright and moral rights for the publications made accessible in the Research Explorer are retained by the authors and/or other copyright owners and it is a condition of accessing publications that users recognise and abide by the legal requirements associated with these rights.

\section{Takedown policy}

If you believe that this document breaches copyright please refer to the University of Manchester's Takedown Procedures [http://man.ac.uk/04Y6Bo] or contact uml.scholarlycommunications@manchester.ac.uk providing relevant details, so we can investigate your claim.

\section{OPEN ACCESS}




\section{(E) Cochrane Library}

Cochrane Database of Systematic Reviews

\section{Compression bandages or stockings versus no compression for treating venous leg ulcers (Protocol)}

Shi C, Dumville JC, Cullum N

Shi C, Dumville JC, Cullum N.

Compression bandages or stockings versus no compression for treating venous leg ulcers.

Cochrane Database of Systematic Reviews 2019, Issue 8. Art. No.: CD013397.

DOI: 10.1002/14651858.CD013397.

www.cochranelibrary.com 


\section{TABLE OF CONTENTS}

HEADER . . . . . . . . . . . . . . . . . . . . . . . . . . . . . . . . . . . . . . . 1

ABSTRACT . . . . . . . . . . . . . . . . . . . . . . . . . . . . . . . . . . . . . . . 1

BACKGROUND . . . . . . . . . . . . . . . . . . . . . . . . . . . . . . . . . . . . 1

OBJECTIVES . . . . . . . . . . . . . . . . . . . . . . . . . . . . . . . . . . . . . .

METHODS . . . . . . . . . . . . . . . . . . . . . . . . . . . . . . . . . . . . . . .

ACKNOWLEDGEMENTS . . . . . . . . . . . . . . . . . . . . . . . . . . . . . . . . . . . . . . . . .

REFERENCES . . . . . . . . . . . . . . . . . . . . . . . . . . . . . . . . . . . . . . . 9

APPENDICES . . . . . . . . . . . . . . . . . . . . . . . . . . . . . . . . . . . . . . . . . . . . . . .

CONTRIBUTIONS OF AUTHORS . . . . . . . . . . . . . . . . . . . . . . . . . . . . . . . . . . . . . . . 16

DECLARATIONS OF INTEREST . . . . . . . . . . . . . . . . . . . . . . . . . . . . . . . . . . . . . . . .

SOURCES OF SUPPORT . . . . . . . . . . . . . . . . . . . . . . . . . . . . . . . . . . . . . . . . . . . . . . . 
[Intervention Protocol]

\title{
Compression bandages or stockings versus no compression for treating venous leg ulcers
}

\author{
Chunhu Shi ${ }^{1}$, Jo C Dumville ${ }^{1}$, Nicky Cullum ${ }^{1}$ \\ ${ }^{1}$ Division of Nursing, Midwifery and Social Work, School of Health Sciences, Faculty of Biology, Medicine and Health, University of \\ Manchester, Manchester Academic Health Science Centre, Manchester, UK
}

Contact address: Chunhu Shi, Division of Nursing, Midwifery and Social Work, School of Health Sciences, Faculty of Biology, Medicine and Health, University of Manchester, Manchester Academic Health Science Centre, Manchester, Greater Manchester, M13 9PL, UK. chunhu.shi@manchester.ac.uk.

Editorial group: Cochrane Wounds Group.

Publication status and date: New, published in Issue 8, 2019.

Citation: Shi C, Dumville JC, Cullum N. Compression bandages or stockings versus no compression for treating venous leg ulcers. Cochrane Database of Systematic Reviews 2019, Issue 8. Art. No.: CD013397. DOI: 10.1002/14651858.CD013397.

Copyright (C) 2019 The Cochrane Collaboration. Published by John Wiley \& Sons, Ltd.

\begin{abstract}
A B S T R A C T
This is a protocol for a Cochrane Review (Intervention). The objectives are as follows:

To assess the effects of using compression bandages or stockings, compared with no compression, on the healing of venous leg ulcers in any setting and population.
\end{abstract}

\section{B A C K G R O U N D}

\section{Description of the condition}

Leg ulcers are open skin wounds on the lower leg (typically below the knee and mainly above the ankle) that can last weeks, months or even years. They occur as a consequence of arterial or venous insufficiency, or both. Less frequently, chronic leg ulceration may occur due to some other disease, such as rheumatoid disease or rarer conditions (Bafaraj 2014). Most leg ulcers are the result of venous disease (Jockenhöfer 2014), where blood flow in the veins can be impaired by vein damage, obstruction and calf muscle pump failure (Eberhardt 2014). These problems mean that blood no longer returns efficiently from the legs to the heart and the pressure within the veins rises (Ghauri 2010). The precise chain of events that links high venous pressures with skin breakdown and subsequent chronic wounds is not fully understood.
Leg ulcers of mixed aetiology (those that have more than one cause) usually involve a combination of venous and arterial disease. Open skin ulceration that is due solely to limb ischaemia (a lack of oxygen reaching the leg tissues, i.e. arterial disease) is less common.

Current, accurate estimates of the proportion of leg ulcers due to specific aetiologies can be hard to identify because most studies do not differentiate between venous, arterial or mixed aetiologies of leg ulceration, or do so for each limb but not for each patient (Moffatt 2004; Srinivasaiah 2007; Vowden 2009a). Two pointprevalence surveys undertaken in the north of England estimated that venous ulceration has a prevalence of approximately 0.30 cases per 1000 population in the UK (Cullum 2016; Gray 2018), whilst mixed arterial/venous leg ulceration has a prevalence of 0.11 per 1000 (Cullum 2016). A review of studies of the prevalence of complex wounds suggested that there are limited high-quality data for estimating the burden of venous leg ulceration in lower- and middle-income countries (Cullum 2016).

Compression bandages or stockings versus no compression for treating venous leg ulcers (Protocol)

Copyright ( 2019 The Cochrane Collaboration. Published by John Wiley \& Sons, Ltd. 
The making of a differential diagnosis of the underlying aetiology of a specific leg ulcer is made by taking a clinical history, physical examination, laboratory tests and other assessments (SIGN 2010). Typically, the latter includes an assessment of the arterial blood supply to the leg using the ankle-brachial pressure index (ABPI), measured using a hand-held Doppler ultrasound scanner.

Leg ulcers are associated with considerable cost to patients and to healthcare providers. Two systematic reviews summarised the literature on health-related quality of life in patients with leg ulcers (Herber 2007; Persoon 2004). Both included qualitative and quantitative evaluations and reported that the presence of leg ulceration was associated with pain, restriction of work and leisure activities, impaired mobility, sleep disturbance, reduced psychological well-being and social isolation. Recent research suggests that people with complex wounds, including those with venous leg ulcers, commonly see complete wound healing as the most important outcome (Cullum 2016). Leg ulceration is typically a long-term condition, with periods of healing followed by recurrence stretching over years.

The financial cost of treating a person with an open venous leg ulcer in the UK was estimated at approximately GBP 1700 per year at 2012 prices (Ashby 2014). Nursing time comprises a large part of ulcer treatment costs. A study in Bradford, UK (population approximately 500,000) estimated that for the financial year 2006 to 2007, GBP 1.69 million was spent on dressings and compression bandages and GBP 3.08 million was spent on nursing time (estimates derived from resource use data for all wound types, not just venous leg ulcers) (Vowden 2009b). In the USA the estimated healthcare cost for people with venous leg ulcers was USD 14.9 billion (2012 prices, all payers including Medicare, private, selfinsured) (Rice 2014). In four community wound care clinics in Queensland, Australia, the mean weekly cost for each patient with a venous leg ulcer was estimated as AUD 294.72 at 2016/2017 prices for patients receiving guideline-based care (i.e. with at least one ABPI and compression therapy) (Barnsbee 2019).

\section{Description of the intervention}

The first-line treatment for venous leg ulcers is compression therapy in the form of bandages, stockings or other devices (Partsch 2015). This application of external pressure around the lower leg assists venous return (blood flow back to the heart) and reduces venous reflux. This review focuses on the effects of compression delivered by bandages and stockings.

\section{Compression bandages}

Bandages are categorised as retention, support or compression, depending on their performance in standardised laboratory tests. Compression bandages are further divided according to the amount of force required to extend them and, therefore, the level of compression that they can apply to a limb. Furthermore, the laboratory performance of a bandage may not reflect its performance in clinical use, as this depends upon operator training and application technique (specifically, whether the bandage is applied as a spiral or figure-of-eight, how many layers are applied and the amount of extension used). Compression systems commonly used for venous leg ulcers are listed below (Thomas 1995).

- Class 3a: light-compression bandages; apply $14 \mathrm{mmHg}$ to $17 \mathrm{mmHg}$ pressure at the ankle when applied in a simple spiral, e.g. Elset $^{\circledR}$ (Mölnlycke).

- Class 3b: moderate-compression bandages; apply 18 $\mathrm{mmHg}$ to $24 \mathrm{mmHg}$ pressure at the ankle when applied as a simple spiral, e.g. Velkomp (Datt Mediproducts Pvt. Limited).

- Class 3c: high-compression bandages; apply $25 \mathrm{mmHg}$ to $35 \mathrm{mmHg}$ pressure at the ankle when applied as a simple spiral, e.g. Setopress (Mölnlycke), and Elodur ${ }^{\circledR}$ forte (BSN Medical).

- Class 3d: extra-high-compression bandages; apply up to 60 $\mathrm{mmHg}$ pressure at the ankle when applied as a simple spiral.

\section{Classification of compression systems}

In 2008 a new compression bandage classification system was proposed based on components rather than the number of 'layers' of bandage (Partsch 2008). The Partsch group recommended that the components of compression, such as orthopaedic wool, crepe bandage or cohesive elastic bandage, should be described. Other recommended classification criteria included sub-bandage pressure (measured in the medial gaiter area with the patient supine) and the elastic property of the overall compression system. The following are examples of multi-component bandage systems (listed for illustrative purposes only; not intended as practice recommendations).

- Short stretch/inelastic systems: orthopaedic padding plus one or two rolls of short stretch bandage (SSB).

- Inelastic paste systems: paste bandage plus support bandage, e.g. Setocrepe ${ }^{\circledR}$ (Mölnlycke).

- Two-component bandage systems: orthopaedic padding plus elastic bandage, e.g. $3 \mathrm{M}^{T M}$ Coban $^{T M} 2$ Compression System.

- Four-component bandage systems: orthopaedic padding plus support bandage (crepe) plus class 3a bandage, e.g. PROFORE ${ }^{T M}$ compression system (Smith \& Nephew).

The earliest Cochrane Review of compression for venous leg ulcers (Cullum 2000) defined different compression systems in terms of the number of layers whereas, in line with the recommendations of the consensus group outlined above, subsequent versions refer to components. Nonetheless, where a trial treatment is the original Charing Cross four-layer bandage, or a close variant of it, we have continued to use the term 'four-layer bandage' (4LB), as this is an internationally recognised bandage system.

It is more difficult to classify different compression systems in relation to sub-bandage pressures since, in general, this information is not available from clinical trial reports. In order to gain fur- 
ther insights into the optimal way to classify different compression systems, we consulted with experts in wound management and invited them to complete a survey. The survey listed different types of compression against various classifications and respondents were asked to provide the best choice of classification in their opinion. In addition, free-text comments were invited. As far as possible, the information gleaned from this exercise will be used in classifying and grouping different types of compression therapy in this review.

\section{Compression stockings}

Compression stockings (or hosiery) can be used to treat open ulceration and to reduce the risk of recurrence post-healing. Stockings are classified in a similar way to bandages, according to the level of compression applied to the limb. Importantly, stockings are subject to less operator variability than bandages.

- Class 1: light-support stockings; provide $14 \mathrm{mmHg}$ to 17 $\mathrm{mmHg}$ pressure at the ankle. Used to treat varicose veins.

- Class 2: medium-support stockings; provide $18 \mathrm{mmHg}$ to $24 \mathrm{mmHg}$ pressure at the ankle. Used to treat more severe varicosities, and to prevent venous leg ulcers.

- Class 3: strong-support stockings; provide $25 \mathrm{mmHg}$ to 35 $\mathrm{mmHg}$ pressure at the ankle. Used to treat severe chronic hypertension and severe varicose veins, and to prevent venous leg ulcers.

Alongside compression, wound dressings are almost always applied to open ulcers. Dressings protect the surface of the ulcer, absorb exudate and can be antimicrobial. A series of reviews has addressed the comparative effectiveness of dressings for venous ulcers (Norman 2018; O’Meara 2013; O’Meara 2015). Other treatments for venous leg ulcers include venous surgery (removal of incompetent superficial veins (Gohel 2018)) and drugs such as pentoxifylline (Jull 2012).

\section{How the intervention might work}

Generally, compression therapy is thought to work by applying an external pressure to the leg which assists venous return (blood flow back to the heart) and reduces venous reflux (Woo 2013). Partsch has suggested that compression:

- reduces oedema by reducing capillary filtration, moving fluid from compressed tissues to non-compressed tissues and improving lymphatic drainage (Partsch 2011); and

- reduces the pressure in the veins by increasing venous blood flow and reducing venous pooling (Partsch 2011).

The use of compression to treat venous leg ulcers is not without risk. Whilst Mosti has suggested that compression may increase arterial inflow (Mosti 2012), if the applied pressure exceeds the local arterial perfusion pressure then arterial inflow will be reduced, which risks ischaemia.
National clinical guidelines in the UK and USA recommend that all patients presenting with a leg ulcer be screened for arterial disease using Doppler-aided measurement of the ABPI (Bolton 2014; SIGN 2010). Clinically significant arterial disease is often defined as an ABPI of below 0.8. Patients with venous leg ulceration who have an ABPI of between 0.5 and 0.8 may be eligible to receive modified (reduced) compression (Moffatt 2007).

\section{Why it is important to do this review}

Venous leg ulcers have a large impact on people's lives and incur high costs to health services; and compression therapy is currently the first line treatment. Since the publication of the original Cochrane Review of compression bandages and stockings for venous leg ulcers (Cullum 2000), the number of relevant randomised controlled trials (RCTs) has more than doubled; the range of compression modalities has increased; and the classification of compression modalities has been refined. In order to offer up-todate evidence for decision makers, we have decided to update the previous version of this review, O'Meara 2012, and to split it into separate reviews of different compression modalities. We will then bring the results together into an overview, which will incorporate a network meta-analysis (Salanti 2012), in order to rank the different treatments on their individual probabilities of being the most effective compression modalities for healing venous leg ulcers. This particular review provides evidence about the comparison of compression bandages or stockings versus no compression.

\section{O B J E C T I VES}

To assess the effects of using compression bandages or stockings, compared with no compression, on the healing of venous leg ulcers in any setting and population.

\section{METHODS}

\section{Criteria for considering studies for this review}

\section{Types of studies}

Published and unpublished RCTs, including cluster-RCTs, will be eligible for inclusion irrespective of language of report. Studies which used quasi-randomised methods to allocate treatment (e.g. alternation or odd/even case numbers) will be excluded. Trials will be included if the application of compression is the only systematic difference between study arms. 


\section{Types of participants}

Randomised controlled trials which recruited people of any age with venous leg ulceration (which may also be described as 'stasis' or 'varicose' ulceration) in any care setting will be eligible for inclusion. As the method of diagnosis of venous ulceration can vary between studies, we will apply no standardised definition, but each study must refer to the use of compression for venous ulcers. We will include studies that recruited participants with a variety of wound types including venous leg ulcers if: a) the allocation of participants was stratified by wound type and included 'venous leg ulcer' as a group and results were presented (or available from the study authors) separately for this group; or b) studies included participants with non-venous leg ulcers, but these made up a maximum of $25 \%$ of the total study population and we will assume that any treatment effect applies to people with venous ulcers. RCTs which only recruited people with non-venous leg ulcers (e.g. arterial, or mixed) will be excluded from the review.

\section{Types of interventions}

We will include trials which compared the use of any compression bandage or stocking or any combination of compression with no compression (e.g. standard care, simple retention bandages, dressings alone) in participants with venous leg ulcers. We will exclude trials where intermittent pneumatic compression is the mode of compression being evaluated, as this is the focus of another Cochrane Review (Nelson 2014).

\section{Types of outcome measures}

\section{Assessment of outcomes at different follow-up periods}

We will group outcome data using the following time categories; we will use our judgement to decide whether statistical pooling within these categories is appropriate.

- Short term: up to eight weeks.

- Medium term: between eight and 24 weeks.

- Long term: more than 24 weeks.

Where relevant, we will report outcomes at the latest time point available (assumed to be length of follow-up, if not specified) and the time point specified in the methods as being of primary interest (if this is different from the latest time point available).

\section{Primary outcomes}

The primary effectiveness outcome for this review is ulcer healing. Trialists use a range of different methods for measuring and reporting this outcome. RCTs that report one or more of the following will be considered as providing the most relevant and rigorous measures of wound healing.
- Time to complete wound healing (correctly analysed using survival, time-to-event approaches or median time to healing, if it was clear that all wounds were healed at follow-up).

- Proportion of wounds completely healed during follow-up (frequency of complete healing).

We will use the study authors' definitions of complete wound healing, and will report these where possible. Where both the complete wound healing outcomes above are reported for a study, we will present both and give precedence to time-to-healing in our interpretation where possible.

The primary safety outcome for the review will be all reported adverse events. Where reported, and a clear methodology for the collection of adverse event data has been provided, we will extract data for all serious adverse events and all non-serious adverse events. The methodology should make it clear whether events were reported at the participant level or, if multiple events/people were reported, that an appropriate adjustment was made for data clustering.

\section{Secondary outcomes}

- Participant health-related quality of life/health status: measured using a standardised generic questionnaire such as EQ5D (Herdman 2011), SF-36 (Ware 1992), SF-12 (Ware 1996) or SF-6 (Craig 2013), or wound-specific questionnaires such as the Cardiff Wound Impact Schedule (Price 2004). We will not include ad hoc measures of quality-of-life that are not likely to be validated and would not be common to multiple trials.

- Cost effectiveness: within-trial cost-effectiveness analysis comparing mean differences in effects with mean cost differences between the two arms. Data extracted could be incremental mean cost per incremental gain in benefit (incremental costeffectiveness ratio (ICER)).

- Ulcer recurrence: measured as time to recurrence or the proportion of recurrence events in a specific time period, or both.

- Mean pain score (including pain at dressing change): measured as a continuous outcome using a validated scale such as a visual analogue scale (VAS) or other recognised measurement instrument.

- Participant adherence to compression treatment: measured as time on allocated compression treatment, or proportion of participants changing from compression treatment.

\section{Search methods for identification of studies}

\section{Electronic searches}

We will search the following databases to retrieve reports of relevant clinical trials:

- the Cochrane Wounds Specialised Register (to present); 
- the Cochrane Central Register of Controlled Trials

(CENTRAL) in the Cochrane Library (to latest issue);

- Ovid MEDLINE including In-Process \& Other Non-

Indexed Citations (from 1946 to present);

- Ovid Embase (from 1974 to present); and

- EBSCO CINAHL Plus (Cumulative Index to Nursing and

Allied Health Literature; from 1937 to present).

We have devised a draft search strategy for CENTRAL which is presented in Appendix 1. We will adapt this strategy to search the Cochrane Wounds Specialised Register, Ovid MEDLINE, Ovid Embase and EBSCO CINAHL Plus. We will combine the Ovid MEDLINE search with the Cochrane Highly Sensitive Search Strategy for identifying randomised trials in MEDLINE: sensitivity- and precision-maximising version (2008 revision) (Lefebvre 2011). We will combine the Embase search with the Ovid Embase filter terms developed by the UK Cochrane Centre (Lefebvre 2011). We will combine the CINAHL Plus search with the trial filter developed by the Scottish Intercollegiate Guidelines Network (SIGN 2018). There will be no restrictions of the searches with respect to language, date of publication or study setting.

We will also search the following trials registries for ongoing trials or completed trials that have not been published.

- ClinicalTrials.gov (www.clinicaltrials.gov);

- World Health Organization International Clinical Trials

Registry Platform (ICTRP) (www.who.int/trialsearch).

\section{Searching other resources}

We will attempt to identify other potentially eligible trials or ancillary publications by searching the reference lists of retrieved included trials as well as relevant systematic reviews, meta-analyses, and seven clinical practice guidelines for leg ulcers (Bolton 2014; Franks 2016; Marston 2016; O’Donnell 2014; SIGN 2010; Australian Wound Management Association 2011; Wittens 2015).

\section{Data collection and analysis}

\section{Selection of studies}

Two review authors will independently assess the titles and abstracts of the citations retrieved by the searches for relevance. After this initial assessment, we will obtain full-text copies of all studies considered to be potentially relevant. Two review authors will independently check the full papers for eligibility; disagreements will be resolved by discussion and, where required, the input of a third review author. Where the eligibility of a study is unclear we will attempt to contact study authors. We will record all reasons for exclusion of studies for which we obtain full-text copies. We will complete a PRISMA flowchart to summarise this process (Liberati 2009).
Where studies are reported in multiple publications or reports, we will obtain all publications. Whilst the study will be included only once in the review, obtaining all publications will maximise the amount of data we can extract. We will also examine any relevant retraction statements and errata for information.

\section{Data extraction and management}

We will extract and summarise details of the eligible studies using a data extraction sheet. Two review authors will extract data independently and will resolve disagreements through discussion, consulting a third review author where required. Where data are missing from reports, we will attempt to contact the study authors to obtain this information.

Where possible we will extract the following data:

- country of origin;

- trial design (e.g. parallel, cluster);

- study population, including key related medical histories, diagnosis methods, the aetiology of leg ulcers (e.g. postthrombotic syndrome, varicose veins, chronic venous reflux), the onset or recurrence of leg ulcers, and the location of leg ulcers;

- care setting;

- eligibility criteria and key baseline participant data (total number of participants, age, sex, duration of leg ulcers, baseline leg ulcer area);

- details of the interventions, including compression devices used, and duration of interventions applied;

- descriptions of any co interventions or standard care;

- follow-up period;

- unit of randomisation (e.g. leg ulcer, limb, or participant);

- numbers of participants randomised to each intervention;

- unit of analyses;

- number of ulcers per person;

- primary and secondary outcomes measured;

- data regarding time to complete wound healing: hazard ratio (HR) and its $95 \%$ confidence interval $(\mathrm{CI})$, or any data that will allow its calculation (Parmar 1998; Tierney 2007);

- data regarding proportion of wounds completely healed during follow-up: odds ratio (OR) and its $95 \% \mathrm{CI}$, or numbers of participants who have leg ulcers completely healed in each arm, both at the latest time point and (if different) at another time specified as of primary interest in the methods section;

- whether a Kaplan Meier plot is displayed;

- missing data rates per arm, and reasons for 'missingness', including the number of people who died;

- publication status of study; and

- source of funding for trial.

\section{Assessment of risk of bias in included studies}

Two review authors will independently assess each included study using the Cochrane tool for assessing risk of bias (Higgins 2011a). 
This tool addresses six specific domains: sequence generation, allocation concealment, blinding, incomplete data, selective outcome reporting, and other issues. In this review we will include unit of analysis issues under the domain of 'other issues', for example where a cluster-randomised trial has been undertaken but analysed at the individual level in the study report. We will assess blinding of participants and personnel, blinding of outcome assessment, and incomplete outcome data for each of the review outcomes separately. We note that blinding of participants and personnel as to whether or not participants have been allocated to compression is impossible and therefore performance bias is a risk. Performance bias may be introduced when awareness of treatment allocation results in deviations from intended interventions and/or differential co-interventions use or care between groups not specified in study protocol which may influence outcomes. We will scrutinise study reports and protocols (where available) to understand if, and how, studies attempted to minimise and document protocol deviations and differential care/co interventions compensated for this: for example, the study protocol might have been used to highlight the need to balance co interventions as well as potentially measuring and reporting this.

We will assess risk of bias for each domains as either: low risk, high risk or unclear risk. Since wound healing is a subjective outcome, unblinded outcome assessment represents a high risk of bias (Hróbjartsson 2012). We will therefore only record open intervention studies with blinded outcome assessment as being at low risk of detection bias.

All disagreements in 'Risk of bias' assessment will be resolved by discussion and, where required, the input of a third review author will be sought. Where possible, when a lack of reported information results in a judgement of unclear risk of bias, we will contact study authors for clarification. We will present our assessment of risk of bias using two 'Risk of bias' summary figures; one will be a summary of bias for each item across all studies, and the second will show a cross-tabulation of each trial by all of the 'Risk of bias' items. We will classify studies with an assessment of high risk of bias for one or more of the following domains as being at high risk of bias overall for the specified outcome: sequence generation, allocation concealment or blinding of outcome assessment.

For trials using cluster randomisation, we will consider the risk of bias in relation to: recruitment bias, baseline imbalance, loss of clusters, incorrect analysis and comparability with individually randomised trials (Higgins 2011a; Eldridge 2016) (Appendix 2).

\section{Measures of treatment effect}

For dichotomous outcomes (e.g. proportion of participants who have wounds completely healed during follow-up), we will calculate the risk ratio (RR) with $95 \%$ CIs. For continuous outcomes we will use the mean difference (MD) with $95 \% \mathrm{CIs}$, for trials that used the same assessment scale. If trials that report continuous data used different assessment scales, we will use the standardised mean difference (SMD) with 95\% CIs.

Time-to-event data (e.g. time to complete wound healing) will be reported as hazard ratios (HRs) where possible, in accordance with the methods described in the Cochrane Handbook for Systematic Reviews of Interventions (Deeks 2011). If studies reporting timeto-event data (e.g. time to healing) do not report a HR, then, when feasible, we will estimate this using other reported outcomes (such as numbers of events) through the application of available statistical methods (Parmar 1998; Tierney 2007). We will only consider median time to healing without survival analysis as a valid outcome if reports specify that all leg ulcers have healed (i.e. if the trial authors treat time-to-healing as a continuous measure as there is no censoring).

\section{Unit of analysis issues}

We will note whether trials present outcomes at the level of the leg ulcer, the limb or the participant and whether there may have been multiple ulcers reported for the same participant. Where trials randomise at the participant level and outcomes are measured at the wound level, e.g. leg ulcer healing, we will treat the participant as the unit of analysis when the number of leg ulcers assessed appears to be equal to the number of participants (e.g. one leg ulcer per person).

A particular unit of analysis issue may occur in trials if randomisation was carried at the participant level, with the allocated treatment used on multiple leg ulcers per participant, but data are presented and analysed per leg ulcer (clustered data). We will note whether data regarding multiple ulcers on a participant were (incorrectly) treated as independent within a study, or were analysed using within-participant analysis methods. If clustered data were incorrectly analysed, we will record this as part of the 'Risk of bias' assessment. For an individually randomised trial, such data on multiple leg ulcers may be collected and analysed:

- only in a proportion of participants (in this case, we will only extract and present relevant data but not treat the trial as a cluster trial to seek for an analysis because the trial incorrectly includes a mixture of individual and clustered data); or

- in all participants (in this case, we will treat the trial as a cluster trial and will incorporate relevant data in meta-analyses if the trial was analysed correctly). Where a cluster trial was not incorrectly analysed, we will record this in the 'Risk of bias' assessment. Where possible we will approximate the correct analyses based on guidance in the Cochrane Handbook for Systematic Reviews of Interventions (Higgins 2011b).

Useful information for approximating the correct analyses include:

- the number of clusters randomised to each arm or the average size of each cluster;

- the outcome data ignoring the cluster design; and

- an estimate of the intracluster correlation coefficient (ICC). 


\section{Dealing with missing data}

It is common for there to be data missing from trial reports. Excluding participants from the analysis post-randomisation or ignoring participants who are lost to follow-up compromises the randomisation and potentially introduces bias into the trial. If it is thought that study authors might be able to provide some missing data, we will attempt to contact them; however, it is likely that data will often be missing because of loss to follow-up.

In individual studies, when data for the proportion of leg ulcers healed are presented, we will assume that randomly assigned participants not included in an analysis had an unhealed leg ulcer at the end of the follow-up period (i.e. they will be considered in the denominator but not in the numerator). We will examine the impact of this assumption through doing a sensitivity analysis (see Sensitivity analysis) in which we will assume participants with missing data had a healed leg ulcer (i.e. they will be included in both the numerator and the denominator). When a trial does not specify participant group numbers before dropout, we will present only complete case data. For the time-to-healing analysis using survival analysis methods, dropouts should be accounted for as censored data. Hence all participants would contribute to the analysis. We acknowledge that such analysis assumes that dropouts are missing at random and there is no pattern of 'missingness'.

We will present data for all categorical secondary outcomes as a complete case analysis. For continuous secondary outcome variables, e.g. length of hospital stay, we will present available data from the study reports/study authors and will not impute missing data. We will calculate measures of variance when these are missing (Deeks 2011) or we will contact study authors, where possible. Where these measures of variation remain unavailable, we will exclude the study from any relevant meta-analyses.

\section{Assessment of heterogeneity}

Assessment of heterogeneity can be a complex, multi-faceted process. Firstly, we will consider clinical and methodological heterogeneity: that is the degree to which the included studies vary in terms of participants' characteristics (e.g. mean age, proportion of participants by sex, methods of diagnosing leg ulcers), interventions (e.g. delivery approaches of compression systems), outcome definitions and other characteristics such as duration of followup. This assessment of clinical and methodological heterogeneity will be supplemented by information regarding statistical heterogeneity. We will assess statistical heterogeneity using the $\mathrm{Chi}^{2}$ test (a significance level of $\mathrm{P}$ value less than 0.10 will be considered to indicate statistically significant heterogeneity) in conjunction with the $\mathrm{I}^{2}$ measure (Higgins 2003). $\mathrm{I}^{2}$ examines the percentage of total variation across RCTs that is due to heterogeneity rather than chance (Higgins 2003). Very broadly, we will consider that $\mathrm{I}^{2}$ values of $25 \%$, or less may not indicate important heterogeneity and values of more than $75 \%$ indicate considerable heterogeneity (Deeks 2011; Higgins 2003).
These statistical tests are recognised to be underpowered and should be only used as an indicator of heterogeneity. Therefore the clinical, methodological and statistical heterogeneity should be considered together for the overall assessment of heterogeneity. Where there is no clinical or statistical heterogeneity, we will use a fixed-effect model. In the absence of clinical heterogeneity and in the presence of some statistical heterogeneity ( $\mathrm{I}^{2}$ over $50 \%$ ), we will use a random-effects model; however, we will not anticipate pooling data across studies where heterogeneity is considerable ( ${ }^{2}$ over $75 \%$ ). Where there is evidence of considerable heterogeneity we will explore this further if required: see Data synthesis.

\section{Assessment of reporting biases}

Reporting biases arise when the dissemination of research findings is influenced by the nature and direction of results. Publication bias is one of a number of possible causes of small-study effects, that is, a tendency for estimates of the intervention effect to be more beneficial in smaller RCTs. Funnel plots allow a visual assessment of whether small-study effects may be present in a meta-analysis. A funnel plot is a simple scatter plot of the intervention effect estimates from individual RCTs against some measure of each trial's size or precision (Sterne 2011). Funnel plots are only informative when there are a substantial number of studies included in an analysis; we will present funnel plots for meta-analyses that include at least 10 RCTs, using Review Manager 5 (RevMan 2014).

\section{Data synthesis}

We will summarise details of included studies in a narrative review according to the comparison between intervention and comparator, the participants, and the outcome measurement including the follow-up duration. We will consider clinical and methodological heterogeneity and will undertake pooling if studies appear appropriately similar in terms of participants, intervention comparison, and outcome assessment including follow-up duration. Where studies are not similar enough for pooling, we will present the results of included studies narratively.

We are unable to pre specify the amount of clinical, methodological and statistical heterogeneity in the included studies but it might be extensive. Thus, we anticipate using a random-effects approach for meta-analysis. Conducting meta-analysis with a fixed-effect model in the presence of even minor heterogeneity may provide overly narrow confidence intervals. We will only use a fixed-effect approach when clinical and methodological heterogeneity is assessed to be minimal, and the assumption that a single underlying treatment effect is being estimated holds. $\mathrm{Chi}^{2}$ and $\mathrm{I}^{2}$ will be used to quantify heterogeneity but will not be used to guide choice of model for meta-analysis. We will exercise caution when meta-analysed data are at risk of small-study effects because a random-effects model may be unsuitable. In this case, or where there are other reasons to question the selection of a fixed-effect or random-effects 
model, we will assess the impact of the approach using sensitivity analyses to compare results from alternate models (Thompson 1999). We will report any evidence that suggests that the use of a particular model might not be robust.

We will produce pooled estimates of the treatment effect using Review Manager 5 (RevMan 2014) and present data using forest plots where possible. For time-to-event data, we will plot (and, if appropriate, pool) estimates of HRs and 95\% CIs as presented in the study reports, using the generic inverse variance method in Review Manager 5 (RevMan 2014). Where time-to-healing is analysed as a continuous measure, but it is not clear if all wounds healed, we will document use of the outcome in the study, but will not summarise or use the data in any meta-analysis.

We will include only the relevant arms where a trial involves multiple arms. If two or more arms in comparison with control are eligible for the same meta-analysis, we will pool data on the two or more arms and compare them with control.

\section{Subgroup analysis and investigation of heterogeneity}

When there appears to be considerable between-study heterogeneity we will attempt to explore the causes using the steps proposed by Cipriani 2013:

- check the data extraction and data entry for errors and possible outlying studies;

- if outliers exist, perform sensitivity analysis by removing them; and

- if heterogeneity is still present, perform subgroup analyses/ meta-regression for study-level characteristics (see below) in order to explain heterogeneity as much as possible (Thompson 1999).

For subgroup analysis/meta-regression, we will consider four study-level characteristics: funding sources (binary: non-for-profit versus other/unclear); overall risk of bias (binary: low and unclear risk of bias versus high risk of bias); study designs (binary: parallel versus other designs); and follow-up duration (continuous).

\section{Sensitivity analysis}

For pooled analyses, where possible, we will undertake sensitivity analyses to explore the impact of the following:

- assuming participants with missing data had a healed leg ulcer (i.e. they will be included in both the numerator and the denominator) followed by the analysis with the assumption that participants with missing data had unhealed leg ulcers;

- removing unpublished data (i.e. abstracts and dissertations) from the analysis; and

- changing effects model (i.e. using random-effects model for the main analysis, followed by a repeated analysis with fixedeffect model).
'Summary of findings' tables and assessment of the quality of the evidence using the GRADE approach

We will present the main, pooled results of the review in 'Summary of findings' tables. These tables present key information concerning the certainty of the evidence, the magnitude of the effects of the interventions examined and the sum of available data for the main outcomes (Schünemann 2011).

We will present the following outcomes in the 'Summary of findings' tables:

- time to complete wound healing when analysed using appropriate survival analysis methods;

- proportion of wounds completely healed during the trial period;

- all reported adverse events;

- participant health-related quality of life/health status;

- cost effectiveness.

We will use the principle of the GRADE approach to assess the certainty of the body of evidence associated with each of the main outcomes for the 'Summary of findings' tables. The GRADE approach defines the certainty of a body of evidence with regard to the extent to which one can be confident that an estimate of effect or association is close to the true quantity of specific interest. The assessment of the certainty of a body of evidence using the GRADE approach involves consideration of within-trial risk of bias (methodological quality), directness of evidence, heterogeneity, precision of effect estimates and risk of publication bias (Schünemann 2011). The certainty of evidence can be assessed as being high, moderate, low or very-low; RCT evidence has the potential to be high certainty.

When making decisions regarding methodological quality, we will downgrade our assessment of the certainty of the evidence only when studies are classed as being at an overall high risk of bias. We will not downgrade for assessments of unclear risk of bias.

In assessing the precision of effect estimates we will follow GRADE guidance using the combination of optimal information size (OIS), and the $95 \%$ CIs of effect estimates:

- if the OIS criterion is not met, downgrade for imprecision, unless the sample size is very large (at least 2000, and perhaps 4000, participants);

- if the OIS criterion is met and the $95 \%$ CI excludes no effect (i.e. the $\mathrm{CI}$ around the RR excludes 1.0), do not downgrade for imprecision; and

- if the OIS criterion is met, and the $95 \%$ CI overlaps no effect (i.e. CI includes RR of 1.0) downgrade for imprecision if the CI fails to exclude important benefit or important harm (i.e. the $95 \%$ CIs include a relative risk reduction or increase of $25 \%$ or more).

For binary outcomes, the OIS will be calculated by the review authors on the basis of a relative risk reduction or increase of between $20 \%$ and $30 \%$, as outlined in the GRADE Handbook and summarised below. 
- Time to wound healing: OIS = 524 participants for a reduction in hazard of time to healing of 25\% (with 100 days' recruitment and 100 days' follow-up: $80 \%$ power; alpha $5 \%$ and median time to healing in control group of 90 days).

- Proportion of wounds healed: OIS = 308 participants for an increased relative risk of wound healing of $25 \%$ ( $80 \%$ power; alpha $5 \%$; proportion healed in control group $=45.08 \%$ ).

- All reported adverse events: OIS $=295$ participants for a decreased relative risk of adverse events of $25 \%$ ( $80 \%$ power; alpha 5\%; proportion reported adverse events in control group $=$ $45 \%)$.

For continuous outcomes, we will use the rule-of-thumb threshold (OIS = 400) suggested by Schünemann 2013.

We will consider downgrading twice for imprecision when, in addition to the rules above, the number of outcome events is considered to be low.

When assessing the remaining domains, we will follow GRADE guidance (Schünemann 2013). Where data are not pooled we will present GRADE assessments for the above outcomes narratively in a 'Summary of findings' table (Murad 2017).

\section{ACKNOWLEDGEMENTS}

The authors are grateful to the following peer reviewers: Liz McInnes, Janet Gunderson, Una Adderley and Clifford Richardson. Thanks are also due to Jessica Sharp for copy-editing this protocol.

Elements of this Methods section are based on the standard Cochrane Wounds protocol template.

\section{REFERE N C E S}

\section{Additional references}

\section{Ashby 2014}

Ashby RL, Gabe R, Ali S, Saramago P, Chuang LH, Adderley U, et al. VenUS IV (Venous leg Ulcer Study IV) compression hosiery compared with compression bandaging in the treatment of venous leg ulcers: a randomised controlled trial, mixed-treatment comparison and decisionanalytic model. Health Technology Assessment 2014;18(57): 1-293, v-vi. [PUBMED: 25242076]

Australian Wound Management Association 2011 Australian Wound Management Association. New Zealand Wound Care Society. Australian and New Zealand clinical practice guideline for prevention and management of venous leg ulcers. Osborne Park, 2011.

Bafaraj 2014

Bafaraj MG, Cesko E, Weindorf M, Dissemond J.

Chronic leg ulcers as a rare cause for the first diagnosis of epidermolysis bullosa dystrophica. International Wound Journal 2014;11(3):274-7. [PUBMED: 22974048]

\section{Barnsbee 2019}

Barnsbee L, Cheng Q, Tulleners R, Lee X, Brain D, Pacella R. Measuring costs and quality of life for venous leg ulcers. International Wound Journal 2019;16(1):112-21. [PUBMED: 30289621]

\section{Bolton 2014}

Bolton LL, Girolami S, Corbett L, van Rijswijk L. Venous and pressure ulcer guidelines. Ostomy/Wound Management 2014;60(11):24-66. [PUBMED: 25380098]

Cipriani 2013

Cipriani A, Higgins JP, Geddes JR, Salanti G. Conceptual and technical challenges in network meta-analysis. Annals of Internal Medicine 2013;159(2):130-7. [PUBMED: 23856683]
Craig 2013

Craig BM, Pickard AS, Stolk E, Brazier JE. US valuation of the SF-6D. Medical Decision Making: an International Journal of the Society for Medical Decision Making 2013;33 (6):793-803. [PUBMED: 23629865]

\section{Cullum 2000}

Cullum N, Nelson EA, Fletcher AW, Sheldon TA. Compression bandages and stockings for venous leg ulcers. Cochrane Database of Systematic Reviews 2000, Issue 2. DOI: 10.1002/14651858.CD000265; PUBMED: 10796522

\section{Cullum 2016}

Cullum N, Buckley HL, Dumville J, Hall J, Lamb K, Madden MT, et al. Wounds research for patient benefit: a 5 year programme of research. National Institute for Health Research Journals Library 2016. [PUBMED: 27583319]

\section{Deeks 2011}

Deeks J, Higgins J, Altman D. Chapter 9: Analysing data and undertaking meta-analyses. In: Higgins JP, Green $S$ editor(s). Cochrane Handbook for Systematic Reviews of Interventions Version 5.1.0 (updated March 2011). The Cochrane Collaboration, 2011. Available from handbook5-1.cochrane.org.

Eberhardt 2014

Eberhardt RT, Raffetto JD. Chronic venous insufficiency. Circulation 2014;130(4):333-46. [PUBMED: 25047584]

Eldridge 2016

Eldridge S, Campbell M, Campbell M, Dahota A, Giraudeau B, Higgins J, et al. Revised Cochrane risk of bias tool for randomized trials (RoB 2.0) Additional considerations for cluster-randomized trials. https:// sites.google.com/site/riskofbiastool/welcome/rob-2-0-tool/ archive-rob-2-0-cluster-randomized-trials-2016 (accessed 24 June 2019). 


\section{Franks 2016}

Franks PJ, Barker J, Collier M, Gethin G, Haesler E, Jawien A, et al. Management of patients with venous leg ulcer: challenges and current best practice. Journal of Wound Care 2016;25(Suppl 1):1-67. [PUBMED: 27292202]

Ghauri 2010

Ghauri AS, Nyamekye IK. Leg ulceration: the importance of treating the underlying pathophysiology. Phlebology/ Venous Forum of the Royal Society of Medicine 2010;25(Suppl 1):42-51. [PUBMED: 20870819]

\section{Gohel 2018}

Gohel MS, Heatley F, Liu X, Bradbury A, Bulbulia R, Cullum N, et al. A randomized trial of early endovenous ablation in venous ulceration. The New England Journal of Medicine 2018;378(22):2105-14. [PUBMED: 29688123]

\section{Gray 2018}

Gray TA, Rhodes S, Atkinson RA, Rothwell K, Wilson P, Dumville JC, et al. Opportunities for better value wound care: a multiservice, cross-sectional survey of complex wounds and their care in a UK community population. BMJ Open 2018;22(8):e019440. [PUBMED: 29572395]

\section{Herber 2007}

Herber OR, Schnepp W, Rieger MA. A systematic review on the impact of leg ulceration on patients' quality of life. Health and Quality of Life Outcomes 2007;5:44. [PUBMED: 17651490]

\section{Herdman 2011}

Herdman M, Gudex C, Lloyd A, Janssen M, Kind P, Parkin $\mathrm{D}$, et al. Development and preliminary testing of the new five-level version of EQ-5D (EQ-5D-5L). Quality of Life Research : an International Journal of Quality of Life Aspects of Treatment, Care and Rehabilitation 2011;21(10):1727-36. [PUBMED: 21479777]

\section{Higgins 2003}

Higgins JP, Thompson SG, Deeks JJ, Altman DG. Measuring inconsistency in meta-analyses. BMJ 2003;327 (7414):557-60. [PUBMED: 12958120]

\section{Higgins 2011a}

Higgins JPT, Altman DG, Sterne J. Chapter 8: Assessing risk of bias in included studies. In: Higgins JPT, Green S, (editors). Cochrane Handbook for Systematic Reviews of Interventions Version 5.1.0 (updated March 2011). The Cochrane Collaboration, 2011a. Available from: handbook-5-1.cochrane.org.

\section{Higgins 2011b}

Higgins JPT, Deeks JJ, Altman DG. Chapter 16: Special topics in statistics. In: Higgins JPT, Green S, (editors). Cochrane Handbook for Systematic Reviews of Interventions Version 5.1.0 (updated March 2011). The Cochrane Collaboration, 2011b. Available from: handbook-5-1.cochrane.org.

\section{Hróbjartsson 2012}

Hróbjartsson A, Thomsen AS, Emanuelsson F, Tendal B, Hilden J, Boutron I, et al. Observer bias in randomised clinical trials with binary outcomes: systematic review of trials with both blinded and non-blinded outcome assessors. BMJ 2012;344:e1119. [PUBMED: 22371859]

Jockenhöfer 2014

Jockenhöfer F, Gollnick H, Herberger K, Isbary G, Renner R, Stucker M, et al. Aetiology, comorbidities and cofactors of chronic leg ulcers: retrospective evaluation of 1000 patients from 10 specialised dermatological wound care centers in Germany. International Wound Journal 2016;13 (5):821-8. [PUBMED: 25483380]

\section{Jull 2012}

Jull AB, Arroll B, Parag V, Waters J. Pentoxifylline for treating venous leg ulcers. Cochrane Database of Systematic Reviews 2012, Issue 12. DOI: 10.1002/ 14651858.CD001733.pub3; CD001733; PUBMED: 23235582

Lefebvre 2011

Lefebvre C, Manheimer E, Glanville J. Chapter 6: Searching for studies. In: Higgins JP, Green S, editor(s). Cochrane Handbook for Systematic Reviews of Interventions Version 5.1.0 (updated March 2011). The Cochrane Collaboration, 2011. Available from: handbook-5-1.cochrane.org.

\section{Liberati 2009}

Liberati A, Altman DG, Tetzlaff J, Mulrow C, Gotzsche PC, Ioannidis JP, et al. The PRISMA statement for reporting systematic reviews and meta-analyses of studies that evaluate health care interventions: explanation and elaboration. PLOS Medicine 2009;6(7):e1000100. [PUBMED: 19621070]

\section{Marston 2016}

Marston W, Tang J, Kirsner RS, Ennis W. Wound Healing Society 2015 update on guidelines for venous ulcers. Wound Repair and Regeneration 2016;24(1):136-44. [PUBMED: 26663616]

\section{Moffatt 2004}

Moffatt CJ, Franks PJ, Doherty DC, Martin R, Blewett R, Ross F. Prevalence of leg ulceration in a London population. QJM: Monthly Journal of the Association of Physicians 2004; 97(7):431-7. [PUBMED: 15208431]

\section{Moffatt 2007}

Moffatt C (editor). Compression Therapy in Practice. Aberdeen: Wounds-UK Books, 2007.

\section{Mosti 2012}

Mosti G, Iabichella ML, Partsch H. Compression therapy in mixed ulcers increases venous output and arterial perfusion. Journal of Vascular Surgery 2012;55(1):122-8. [PUBMED: 21944912]

Murad 2017

Murad MH, Mustafa RA, Schünemann HJ, Sultan S, Santesso N. Rating the certainty in evidence in the absence of a single estimate of effect. Evidence-Based Medicine 2017; 22(3):85-7. [PUBMED: 28320705]

\section{Nelson 2014}

Nelson EA, Hillman A, Thomas K. Intermittent pneumatic compression for treating venous leg ulcers. Cochrane Database of Systematic Reviews 2014, Issue 5. DOI: 10.1002/14651858.CD001899.pub4 


\section{Norman 2018}

Norman G, Westby MJ, Rithalia AD, Stubbs N, Soares MO, Dumville JC. Dressings and topical agents for treating venous leg ulcers. Cochrane Database of Systematic Reviews 2018, Issue 6. DOI: 10.1002/14651858.CD012583.pub2

\section{O’Donnell 2014}

O'Donnell TF Jr, Passman MA, Marston WA, Ennis WJ, Dalsing M, Kistner RL, et al. Management of venous leg ulcers: clinical practice guidelines of the Society for Vascular Surgery ${ }^{\circledR}$ and the American Venous Forum. Journal of Vascular Surgery 2014;60(2 Suppl):3S-59S. [PUBMED: 24974070]

\section{O'Meara 2012}

O'Meara S, Cullum N, Nelson EA, Dumville JC. Compression for venous leg ulcers. Cochrane Database of Systematic Reviews 2012, Issue 11. DOI: 10.1002/ 14651858.CD000265.pub3

\section{O'Meara 2013}

O’Meara S, Martyn-St James M. Foam dressings for venous leg ulcers. Cochrane Database of Systematic Reviews 2013, Issue 5. DOI: 10.1002/14651858.CD009907.pub2

\section{O'Meara 2015}

O’Meara S, Martyn-St James M, Adderley UJ. Alginate dressings for venous leg ulcers. Cochrane Database of Systematic Reviews 2015, Issue 8. DOI: 10.1002/ 14651858.CD010182.pub3; PUBMED: 26286189

\section{Parmar 1998}

Parmar MK, Torri V, Stewart L. Extracting summary statistics to perform meta-analyses of the published literature for survival endpoints. Statistics in Medicine 1998;17(24): 2815-34. [PUBMED: 9921604]

\section{Partsch 2008}

Partsch H, Clark M, Mosti G, Steinlechner E, Schuren J, Abel M, et al. Classification of compression bandages: practical aspects. Dermatologic Surgery 2008;34(5):600-9. [PUBMED: 18261106]

\section{Partsch 2011}

Partsch H. Chapter 6: Use of compression therapy. In: Goldman MP, Guex JJ, Weiss RA editor(s). Sclerotherapy. 5th Edition. Edinburgh: W.B. Saunders, 2011:123-55.

\section{Partsch 2015}

Partsch H, Mortimer P. Compression for leg wounds. The British Journal of Dermatology 2015;173(2):359-69. [PUBMED: 26094638]

\section{Persoon 2004}

Persoon A, Heinen MM, van der Vleuten CJ, de Rooij MJ, van de Kerkhof PC, van Achterberg T. Leg ulcers: a review of their impact on daily life. Journal of Clinical Nursing 2004;13(3):341-54. [PUBMED: 15009337]

\section{Price 2004}

Price P, Harding K. Cardiff Wound Impact Schedule: the development of a condition-specific questionnaire to assess health-related quality of life in patients with chronic wounds of the lower limb. International Wound Journal 2004;1(1): 10-17. [PUBMED: 16722893]

\section{RevMan 2014 [Computer program]}

The Nordic Cochrane Centre, The Cochrane Collaboration. Review Manager (RevMan). Version 5.3. Copenhagen: The Nordic Cochrane Centre, The Cochrane Collaboration, 2014.

Rice 2014

Rice JB, Desai U, Cummings AK, Birnbaum HG, Skornicki M, Parsons N. Burden of venous leg ulcers in the United States. Journal of Medical Economics 2014;17(5):347-56. [PUBMED: 24625244]

\section{Salanti 2012}

Salanti G. Indirect and mixed-treatment comparison, network, or multiple-treatments meta-analysis: many names, many benefits, many concerns for the next generation evidence synthesis tool. Research Synthesis Methods 2012;3(2):80-97. [PUBMED: 26062083]

\section{Schünemann 2011}

Schünemann HJ, Oxman AD, Higgins JPT, Vist GE, Glasziou P, Guyatt GH. Chapter 11: Presenting results and 'Summary of findings' tables. In: Higgins JPT, Green S, (editors). Cochrane Handbook for Systematic Reviews of Interventions Version 5.1.0 (updated March 2011). The Cochrane Collaboration, 2011. Available from handbook5-1.cochrane.org.

\section{Schünemann 2013}

Schünemann H, Broż ek J, Guyatt G, Oxman A, editor (s). Handbook for grading the quality of evidence and the strength of recommendations using the GRADE approach (updated October 2013). GRADE Working Group, 2013. Available from guidelinedevelopment.org/handbook.

\section{SIGN 2010}

Scottish Intercollegiate Guidelines Network, Healthcare Improvement Scotland. SIGN Guideline 120: Management of chronic venous leg ulcers. https://www.sign.ac.uk/assets/ sign120.pdf (accessed 24 June 2019).

\section{SIGN 2018}

Scottish Intercollegiate Guidelines Network (SIGN). Search filters. sign.ac.uk/search-filters.html (accessed 22 October 2018).

\section{Srinivasaiah 2007}

Srinivasaiah N, Dugdall H, Barrett S, Drew PJ. A point prevalence survey of wounds in north-east England. Journal of Wound Care 2007;16(10):413-6, 418-9. [PUBMED: 18065016]

\section{Sterne 2011}

Sterne JAC, Egger M, Moher D. Chapter 10: Addressing reporting biases. In: Higgins JP, Green S, editor (s). Cochrane Handbook for Systematic Reviews of Interventions Version 5.1.0 (updated March 2011). The Cochrane Collaboration, 2011. Available from handbook5-1.cochrane.org.

\section{Thomas 1995}

Thomas S. Compression bandages. Cullum N, Roe BH (editors), Leg ulcers: nursing management. Harrow: Scutari, 1995:63-74. 
Thompson 1999

Thompson SG, Sharp SJ. Explaining heterogeneity in metaanalysis: a comparison of methods. Statistics in Medicine 1999;18(20):2693-708. [PUBMED: 10521860]

Tierney 2007

Tierney JF, Stewart LA, Ghersi D, Burdett S, Sydes MR. Practical methods for incorporating summary time-to-event data into meta-analysis. Trials 2007;8:16. [PUBMED: $17555582]$

Vowden 2009a

Vowden KR, Vowden P. The prevalence, management and outcome for acute wounds identified in a wound care survey within one English health care district. Journal of Tissue Viability 2009;18(1):7-12. [PUBMED: 19185788]

Vowden 2009b

Vowden K, Vowden P, Posnett J. The resource costs of wound care in Bradford and Airedale primary care trust in the UK. Journal of Wound Care 2009;18(3):93-4, 96-8. [PUBMED: 19247229]

Ware 1992

Ware JE Jr, Sherbourne CD. The MOS 36-item short-form health survey (SF-36). I. Conceptual framework and item selection. Medical Care 1992;30(6):473-83. [PUBMED: 1593914]

Ware 1996

Ware J Jr, Kosinski M, Keller SD. A 12-Item Short-Form Health Survey: construction of scales and preliminary tests of reliability and validity. Medical Care 1996;34(3):220-33. [PUBMED: 8628042]

Wittens 2015

Wittens C, Davies AH, Bækgaard N, Broholm R, Cavezzi A, Chastanet $S$, et al. Management of chronic venous disease: clinical practice guidelines of the European Society for Vascular Surgery (ESVS). European Journal of Vascular and Endovascular Surgery 2015;49(6):678-737. [PUBMED: 25920631]

Woo 2013

Woo KY, Alavi A, Evans R, Despatis M, Allen J.

New advances in compression therapy for venous leg ulcers. Surgical Technology International 2013;23:61-8. [PUBMED: 24081848]

* Indicates the major publication for the study

\section{A P P E N D I C E S}

\section{Appendix I. The Cochrane Central Register of Controlled Trials (CENTRAL) provisional search strategy}

\#1 MeSH descriptor: [Leg Ulcer] explode all trees

\#2 ((varicose next ulcer*) or (venous next ulcer*) or (leg next ulcer*) or (stasis next ulcer*) or (crural next ulcer*) or (ulcus next cruris) or (ulcer* next cruris)):ti,ab,kw

$\# 3 \# 1$ or \#2

\#4 MeSH descriptor: [Compression Bandages] explode all trees

\#5 compression*:ti,ab,kw

\#6 stocking* or hosiery:ti,ab,kw

\#7 sock or socks or tights:ti,ab,kw

\#8 bandag*:ti,ab,kw

\#9 wrapp*:ti,ab,kw

$\# 10 \# 4$ or $\# 5$ or $\# 6$ or $\# 7$ or \#8 or \#9

$\# 11 \# 3$ and \#10 


\section{Appendix 2. Risk of bias}

\section{I 'Risk of bias' assessment (individually randomised controlled trials)}

\section{Was the allocation sequence randomly generated?}

\section{Low risk of bias}

The investigators describe a random component in the sequence generation process such as: referring to a random number table; using a computer random number generator; coin tossing; shuffling cards or envelopes; throwing dice; drawing of lots.

\section{High risk of bias}

The investigators describe a non-random component in the sequence generation process. Usually, the description would involve some systematic, non-random approach, for example: sequence generated by odd or even date of birth; sequence generated by some rule based on date (or day) of admission; sequence generated by some rule based on hospital or clinic record number.

\section{Unclear}

Insufficient information about the sequence generation process to permit judgement of low or high risk of bias.

\section{Was the treatment allocation adequately concealed?}

\section{Low risk of bias}

Participants and investigators enrolling participants could not foresee assignment because one of the following, or an equivalent method, was used to conceal allocation: central allocation (including telephone, web-based and pharmacy-controlled randomisation); sequentially numbered drug containers of identical appearance; sequentially numbered, opaque, sealed envelopes.

\section{High risk of bias}

Participants or investigators enrolling participants could possibly foresee assignments and thus introduce selection bias, such as allocation based on: using an open random allocation schedule (e.g. a list of random numbers); assignment envelopes were used without appropriate safeguards (e.g. if envelopes were unsealed or non opaque or not sequentially numbered); alternation or rotation; date of birth; case record number; any other explicitly unconcealed procedure.

\section{Unclear}

Insufficient information to permit judgement of low or high risk of bias. This is usually the case if the method of concealment is not described or not described in sufficient detail to allow a definite judgement, for example if the use of assignment envelopes is described, but it remains unclear whether envelopes were sequentially numbered, opaque and sealed.

\section{Blinding - was knowledge of the allocated interventions adequately prevented during the study?}

\section{Low risk of bias}

Any one of the following.

- No blinding, but the review authors judge that the outcome and the outcome measurement are not likely to be influenced by lack of blinding.

Compression bandages or stockings versus no compression for treating venous leg ulcers (Protocol) 
- Blinding of participants and key study personnel ensured, and unlikely that the blinding could have been broken.

- Either participants or some key study personnel were not blinded, but outcome assessment was blinded and the non-blinding of others unlikely to introduce bias.

\section{High risk of bias}

Any one of the following.

- No blinding or incomplete blinding, and the outcome or outcome measurement is likely to be influenced by lack of blinding.

- Blinding of key study participants and personnel attempted, but likely that the blinding could have been broken.

- Either participants or some key study personnel were not blinded, and the non-blinding of others likely to introduce bias.

\section{Unclear}

Any one of the following.

- Insufficient information to permit judgement of low or high risk of bias.

- The study did not address this outcome.

\section{Were incomplete outcome data adequately addressed?}

\section{Low risk of bias}

Any one of the following.

- No missing outcome data.

- Reasons for missing outcome data unlikely to be related to true outcome (for survival data, censoring unlikely to be introducing bias).

- Missing outcome data balanced in numbers across intervention groups, with similar reasons for missing data across groups.

- For dichotomous outcome data, the proportion of missing outcomes compared with observed event risk not enough to have a clinically relevant impact on the intervention effect estimate.

- For continuous outcome data, plausible effect size (difference in means or standardised difference in means) among missing outcomes not enough to have a clinically relevant impact on observed effect size.

- Missing data have been imputed using appropriate methods.

\section{High risk of bias}

Any one of the following.

- Reason for missing outcome data likely to be related to true outcome, with either imbalance in numbers or reasons for missing data across intervention groups.

- For dichotomous outcome data, the proportion of missing outcomes compared with observed event risk enough to induce clinically relevant bias in intervention effect estimate.

- For continuous outcome data, plausible effect size (difference in means or standardised difference in means) among missing outcomes enough to induce clinically relevant bias in observed effect size.

- 'As-treated' analysis done with substantial departure of the intervention received from that assigned at randomisation.

- Potentially inappropriate application of simple imputation.

\section{Unclear}

Any one of the following.

- Insufficient reporting of attrition/exclusions to permit judgement of low or high risk of bias (e.g. number randomised not stated, no reasons for missing data provided).

- The study did not address this outcome.

Compression bandages or stockings versus no compression for treating venous leg ulcers (Protocol) 


\section{Low risk of bias}

Any of the following.

- The study protocol is available and all of the study's prespecified (primary and secondary) outcomes that are of interest in the review have been reported in the prespecified way.

- The study protocol is not available but it is clear that the published reports include all expected outcomes, including those that were prespecified (convincing text of this nature may be uncommon).

\section{High risk of bias}

Any one of the following.

- Not all of the study's prespecified primary outcomes have been reported.

- One or more primary outcomes are reported using measurements, analysis methods or subsets of the data (e.g. subscales) that were not prespecified.

- One or more reported primary outcomes were not prespecified (unless clear justification for their reporting is provided, such as an unexpected adverse effect).

- One or more outcomes of interest in the review are reported incompletely so that they cannot be entered in a meta-analysis.

- The study report fails to include results for a key outcome that would be expected to have been reported for such a study.

\section{Unclear}

Insufficient information to permit judgement of low or high risk of bias. It is likely that the majority of studies will fall into this category.

\section{Other sources of potential bias}

\section{Low risk of bias}

The study appears to be free of other sources of bias.

\section{High risk of bias}

There is at least one important risk of bias. For example, the study:

- had a potential source of bias related to the specific study design used; or

- has been claimed to have been fraudulent; or

- had some other problem.

\section{Unclear}

There may be a risk of bias, but there is either:

- insufficient information to assess whether an important risk of bias exists; or

- insufficient rationale or evidence that an identified problem will introduce bias

\section{2 'Risk of bias' assessment (cluster-randomised controlled trials)}

\section{Recruitment bias}

Recruitment bias (or identification bias) is the bias that occurs in cluster-RCTs if the personnel recruiting participants know individuals' allocation, even when the allocation of clusters has been concealed appropriately. The knowledge of the allocation of clusters may lead

Compression bandages or stockings versus no compression for treating venous leg ulcers (Protocol)

Copyright ( 2019 The Cochrane Collaboration. Published by John Wiley \& Sons, Ltd. 
to bias because the individuals' recruitment in cluster trials is often behind the clusters' allocation to different interventions; and the knowledge of allocation can determine whether individuals are recruited selectively.

This bias can be judged through considering the following questions.

- Were all the individual participants identified/recruited before randomisation of clusters?

- Is it likely that selection of participants was affected by knowledge of the intervention?

- Were there baseline imbalances that suggest differential identification or recruitment of individual participants between arms?

\section{Baseline imbalance}

Baseline imbalance between intervention groups can occur due to chance, problems with randomisation, or identification/recruitment bias. The issue of recruitment bias has been considered above.

In terms of study design, the risk of chance baseline imbalance can be reduced by the use of stratified or pair-matched randomisation. Minimisation - an equivalent technique to randomisation - can be used to achieve better balance in cluster characteristics between intervention groups if there is a small number of clusters.

Concern about the influence of baseline imbalance can be reduced if trials report the baseline comparability of clusters, or statistical adjustment for baseline characteristics.

\section{Loss of clusters}

Similar with missing outcome data in individually randomised trials, bias can occur if clusters are completely lost from a cluster trial, and are omitted from the analysis.

The amount of missing data, the reasons for missingness and the way of analysing data given the missingness should be considered in assessing the possibility of bias.

\section{Incorrect analysis}

Data analyses, which do not take the clustering into account, in cluster trials will be incorrect. Such analyses lead to a "unit of analysis error" and over-precise results (too small standard error) and too small P values. Though these analyses will not result in biased estimates of effect, they (if not correctly adjusted) will lead to too much weight allocated to cluster trials in a meta-analysis.

Note that the issue of analysis may not lead to concern any more and will not be considered substantial if approximate methods are used by reviewers to address clustering in data analysis.

\section{Comparability with individually randomised trials}

In the case that a meta-analysis includes, for example, both cluster and individually randomised trials, potential differences in the intervention effects between different trial designs should be considered. This is because the "contamination" of intervention effects may occur in cluster-randomised trials, which would lead to underestimates of effect. The contamination could be known as a "herd effect": i.e. within clusters, individuals' compliance with using an intervention may be enhanced, which in return affects the estimation of effect.

\section{CONTRIBUTIONS OFAUTHORS}

Chunhu Shi: developed the protocol; coordinated the protocol development; contributed to writing and editing the protocol; advised on the protocol; approved the final version of the protocol prior to submission and is guarantor of the protocol.

Jo C Dumville: conceived the review question; produced the first draft of the protocol; contributed to editing the protocol; advised on the protocol and approved the final version of the protocol prior to submission.

Nicky Cullum: conceived the review question; produced the first draft of the protocol; contributed to editing the protocol; advised on the protocol and approved the final version of the protocol prior to submission.

Compression bandages or stockings versus no compression for treating venous leg ulcers (Protocol) 


\section{Contributions of the editorial base}

Tanya Walsh (Editor): edited the protocol; advised on methodology, interpretation and content; approved the final protocol prior to submission.

Gill Rizzello (Managing Editor): coordinated the editorial process; advised on content; edited the protocol. Sophie Bishop (Information Specialist): designed the search strategy and edited the search methods section. Ursula Gonthier (Editorial Assistant): edited the reference section of the protocol.

\section{DECLARATIONSOF INTEREST}

Chunhu Shi: none known.

Jo Dumville: received research funding from the National Institute for Health Research (NIHR) UK for the production of systematic reviews focusing on high-priority Cochrane Reviews in the prevention and treatment of wounds. This research was co-funded by the NIHR Manchester Biomedical Research Centre and partly funded by the National Institute for Health Research Collaboration for Leadership in Applied Health Research and Care (NIHR CLAHRC) Greater Manchester.

Nicky Cullum: received research funding for wounds-related research and systematic reviews from the NIHR for the production of systematic reviews focusing on high-priority Cochrane Reviews in the prevention and treatment of wounds. This research was co-funded by the NIHR Manchester Biomedical Research Centre, and partly funded by the National Institute for Health Research Collaboration for Leadership in Applied Health Research and Care (NIHR CLAHRC) Greater Manchester.

Research grant funding was received in the 1990s from the NHS Research and Development programme and the Health Technology Assessment Programme for systematic reviews on compression, and for two randomised controlled trials of compression. These RCTs will not be eligible for inclusion in this review. The funders had no role in the conduct of this review.

Clifford Richardson (peer reviewer) declares that one of the review authors was the Head of the department in which he works, though he himself is not involved with the wound care research team within this department and has not been involved with the preparation or writing of this proposal.

\section{SOURCES OF SUPPORT}

\section{Internal sources}

- Division of Nursing, Midwifery and Social Work, School of Health Sciences, Faculty of Biology, Medicine and Health, University of Manchester, UK.

\section{External sources}

- National Institute for Health Research (NIHR), UK.

This project was supported by the National Institute for Health Research, via Cochrane Infrastructure funding to Cochrane Wounds. The views expressed are those of the authors and not necessarily those of the NIHR or the Department of Health and Social Care

- NIHR Manchester Biomedical Research Centre (BRC), UK.

This research was co-funded by the NIHR Manchester BRC. The views expressed in this publication are those of the authors and not necessarily those of the NHS, the National Institute for Health Research or the Department of Health and Social Care.

- National Institute for Health Research Collaboration for Leadership in Applied Health Research and Care (NIHR CLAHRC), Greater Manchester, UK.

Nicky Cullum and Jo Dumville's work on this project was partly funded by the NIHR CLAHRC, Greater Manchester. The funder had no role in the decision to publish, or preparation of the manuscript. However, the review may be considered to be affiliated to the work of the NIHR CLAHRC Greater Manchester. The views expressed herein are those of the authors and not necessarily those of the NHS, NIHR or the Department of Health and Social Care.

Compression bandages or stockings versus no compression for treating venous leg ulcers (Protocol) 